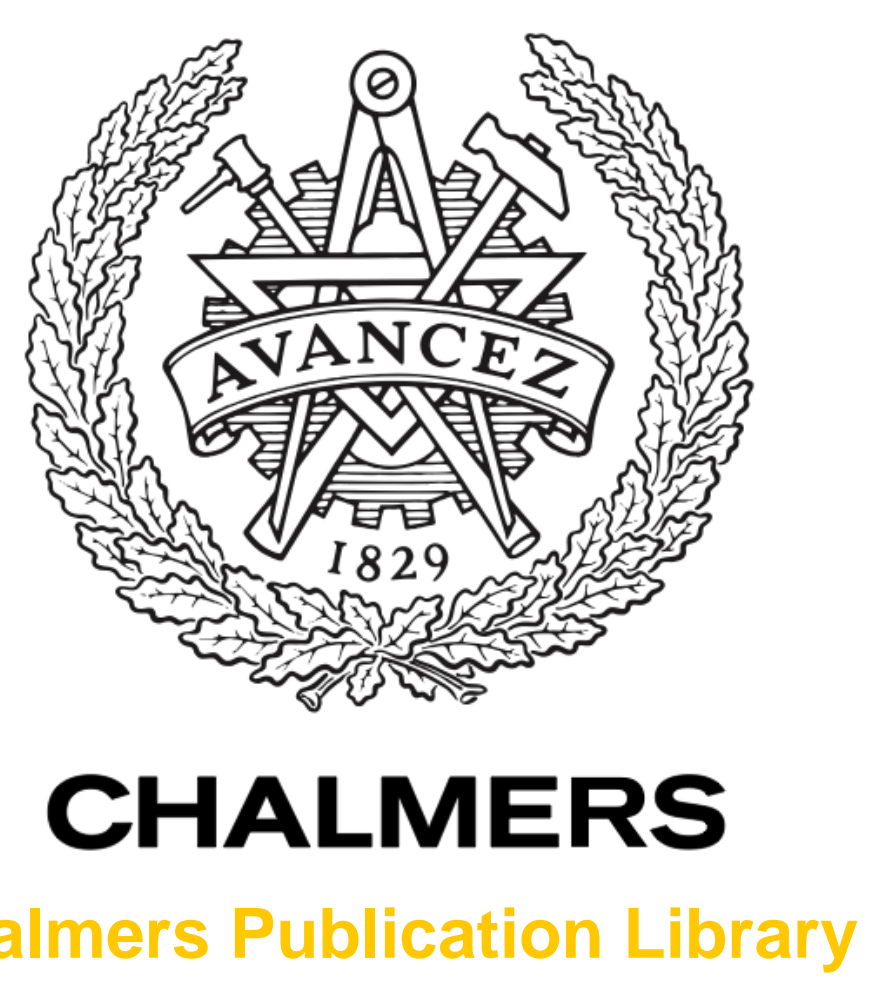

\title{
Comparison of Intersymbol Interference Power Penalties for OOK and 4-PAM in Short-Range Optical Links
}

This document has been downloaded from Chalmers Publication Library (CPL). It is the author's version of a work that was accepted for publication in:

Journal of Lightwave Technology (ISSN: 0733-8724)

Citation for the published paper:

Szczerba, K. ; Westbergh, P. ; Agrell, E. (2013) "Comparison of Intersymbol Interference Power Penalties for OOK and 4-PAM in Short-Range Optical Links". Journal of Lightwave Technology, vol. 31(22), pp. 3525-3534.

http://dx.doi.org/10.1109/JLT.2013.2285468

Downloaded from: http://publications.lib.chalmers.se/publication/187355

Notice: Changes introduced as a result of publishing processes such as copy-editing and formatting may not be reflected in this document. For a definitive version of this work, please refer to the published source. Please note that access to the published version might require a subscription. 


\title{
Comparison of Intersymbol Interference Power Penalties for OOK and 4-PAM in Short-Range Optical Links
}

\author{
Krzysztof Szczerba, Petter Westbergh, Erik Agrell, Magnus Karlsson, Peter A. Andrekson, Anders Larsson.
}

\begin{abstract}
We present results of experimental and theoretical investigations of intersymbol interference in 4-PAM transmission in short-range optical communications links based on the power penalty. A test link comprised of a directly modulated $850 \mathrm{~nm}$ VCSEL with up to $200 \mathrm{~m}$ of multimode fiber and direct detection was used. The link bandwidth was below $10 \mathrm{GHz}$ and the maximum achieved data rate with 4 -PAM was 44 Gbps over $100 \mathrm{~m}$ of fiber. In the same case and at similar sensitivity, only 32 Gbps could be achieved with OOK. If typical forward error correction could be applied, the sensitivity of the 4-PAM system was improved by up to $4 \mathrm{~dB}$, reaching $-10 \mathrm{dBm}$ at $25 \mathrm{Gbps}$.
\end{abstract}

Index Terms-fiber-optical communications, IM/DD, 4-PAM, OOK, VCSEL, MMF, short-range, $850 \mathrm{~nm}$, data communications, interconnects intersymbol interference.

\section{INTRODUCTION}

$\mathbf{T}$ HE development of cloud computing, high performance computing and data centers has created a steady demand for high-capacity short-range optical communications. So far, this demand has been largely answered by the development of fast and low-cost vertical cavity surface emitting lasers (VCSELs) compatible with multi-mode fiber (MMF). Although modal dispersion is the limiting factor in such links, gradedindex MMF, optimized for the $850 \mathrm{~nm}$ wavelength, has been successfully used to reduce the impact of modal dispersion induced intersymbol interference (ISI) and extend the reach in such systems. Currently $10 \mathrm{Gbps}$ links are commercialized and lasers and photoreceivers (mostly with limiting amplifiers) for $25 \mathrm{Gbps}$ at the wavelength of $850 \mathrm{~nm}$ are becoming available. However, with increased bit-rates and recent development of VCSELs capable of operating at $44 \mathrm{Gbps}$ at the wavelength of $850 \mathrm{~nm}$ [1] and earlier at the wavelength of $980 \mathrm{~nm}$ [2], it turns out that the transmission distance in MMF at such high bit-rates is limited by the modal dispersion, if on-off keying (OOK) modulation is used. One possible method to increase the transmission distances is to use VCSELs with narrower spectral width, effectively single or quasi-single mode, for example as in [3], where $20 \mathrm{Gbps}$ transmission over $1 \mathrm{~km}$ of OM4-type MMF was demonstrated with OOK and a quasisingle mode VCSEL operating at $850 \mathrm{~nm}$.

K. Szczerba, P. Westbergh, M. Karlsson, P. A. Andrekson and A. Larsson are with the Department of Microtechnology and Nanoscience, Chalmers University of Technology, Göteborg, Sweden (e-mail: krzysztof.szczerba@chalmers.se).

E. Agrell is with the Department of Signals and Systems, Chalmers University of Technology, Gö, Sweden.

Manuscript received Month Day, Year; revised Month Day, Year.
So far, in all practical applications, OOK modulation is used. Multilevel modulation, with higher spectral efficiency, introduces a new degree of freedom in link design, trading increased required optical power for higher bit rates. Because of cost, latency and power constraints, intensity modulation and direct detection (IM/DD) is appealing in short-range optical networks.

The two main approaches investigated for the purpose of increasing the spectral efficiency in IM/DD links are subcarrier modulation (SCM) and pulse amplitude modulation (PAM). In SCM, a single microwave subcarrier, or multiple subcarriers are first modulated with e.g. quadrature amplitude modulation (QAM) or phase shift keying modulation (PSK). Single-cycle sub-carrier modulation with 16-QAM has been demonstrated in links using VCSELs and multi-mode fibers [4], with the transmitter operating in real-time and the digital processing on the receiver side being implemented off-line. Discrete multi-tone modulation, which is a multiple subcarrier scheme, has been demonstrated at $30 \mathrm{Gbps}$ for the same type of link [5] with off-line processing both in the receiver and in the transmitter. The main advantages of the multiple subcarrier approaches are easier electronic equalization and increased robustness to effects of modal dispersion due to reduced symbol rates. This comes at a cost of worse sensitivity compared to PAM, in terms of optical received power [6], [7] as well as increased complexity and power consumption of the signal processing electronics, whether implemented using analog electronics or digital signal processing.

The complexity of the signal processing electronics is an important limitation in the design of short-range data communication links. Higher complexity of electronics implies increased power consumption and heat generation, which in densely packed data centers increases the cooling difficulties. Therefore, it is difficult to justify the use of subcarrier modulation in such an environment. On the other hand, the PAM is probably the format with the lowest implementation complexity of all multilevel modulation formats. Electronic CMOS circuits for 4-PAM transceivers operating in real-time at bit-rates up to 22 Gbps have already been developed [8], [9]. Multilevel intensity modulation formats, including PAM in particular, were investigated for increasing the reach of $10 \mathrm{Gbps}$ links operating at $1550 \mathrm{~nm}$ wavelength and using standard single-mode fiber in [10]. Electronic pre-distortion was analyzed for extension of the reach of 4-PAM in MMF and demonstrated at $10 \mathrm{Gbps}$ in [11]. In [12], eye diagrams from real-time operation at $32 \mathrm{Gbps}$ with electronic pre-distortion 
were demonstrated. In [13], 4-PAM and OOK were compared in short-range optical links with VCSELs and MMF, with promising results for 4-PAM.

In [14], we have demonstrated 30 Gbps error-free 4-PAM transmission over $200 \mathrm{~m}$ of OM3+ MMF, with a $850 \mathrm{~nm}$ VCSEL. The 4-PAM signal was generated and the bit-error rate (BER) measured, all in real-time. No equalization was used in the receiver, nor was any pre-distortion used in the transmitter in that work. Effects of the ISI for OOK and 4PAM for varying fiber length and fixed data rate were studied theoretically and experimentally in [15]. A similar study of OOK and 4-PAM in electrical on-board interconnects was presented in [16], with a conclusion that OOK with equalization would be a preferred choice. The optical interconnects tend to have different type of frequency response from the microwave transmission lines and thus we do not arrive at the same conclusion as in [16], at least from the point of view of power penalties.

In this work we further study the effects of ISI on 4-PAM data transmission in short-range optical links using VCSELs, MMF and direct detection. In [15], the ISI effects were studied for fixed data rate and increasing fiber length. Conversely, in this work the ISI is studied with fixed length (and thus constant frequency response of the system) but with varied data rates. This gives a clearer comparison to the theoretical results and allows us to evaluate the maximum throughput for each modulation format. The term "throughput" is used, rather than capacity, because channel capacity in the strict meaning is the tight upper bound on the amount of information that can be transmitted reliably through a given channel with any modulation format and any coding. We demonstrate also that the simple model based on Gaussian impulse response and pulse shape, used for the ISI calculation presented in [15], gives a reasonable estimate of the ISI for 4-PAM, but is not without shortcomings. We also look briefly into effects of the forward error correction (FEC) on the sensitivity.

This paper is organized as follows. In Sec. II, basic theory for analytic bit error rate (BER) and ISI penalties is presented. In Sec. III, the experimental setup is described. In Sec. IV, the experimental results are presented and discussed and finally, Sec. V contains concluding remarks.

\section{THEORETICAL PERFORMANCE}

\section{A. Sensitivity}

Multilevel PAM requires increased average received optical power, both at the same bit rate as OOK and at the same symbol rate to reach the same BER. Under assumptions that the noise is additive, white, and stationary (e.g. when the thermal noise is dominating), the optical power penalty for using $M$-level PAM, compared to OOK at the same symbol rate, is

$$
P_{\mathrm{ps}}=10 \log _{10}(M-1),
$$

where $M$ is the number of PAM levels [17]. This means that $4.8 \mathrm{~dB}$ more received optical power is needed for 4-PAM at the same symbol rate as OOK. The penalty is less when the bitrate is kept fixed, because of reduced bandwidth. The optical power penalty for $M$-PAM, relative to OOK at the same bitrate $P_{p b}$, is expressed in $\mathrm{dB}$ as

$$
P_{\mathrm{pb}}=10 \log _{10}\left(\frac{M-1}{\sqrt{\log _{2}(M)}}\right),
$$

according to [10]. In case of 4-PAM, this gives that $3.3 \mathrm{~dB}$ more optical power is required compared to OOK signal at the same bit-rate to reach the same BER. In general, the BER can be expressed as

$$
B E R=\frac{1}{M} \sum_{i=0}^{M-1} \sum_{j=0, j \neq i}^{M-1} \frac{d_{i j}}{\log _{2}(M)} P_{i j},
$$

where $P_{i j}$ is the probability of receiving symbol $j$ when symbol $i$ was transmitted and $d_{i j}$ is the Hamming distance between the labels of symbols $i$ and $j$ [18, Ch. 4.2]. Assuming Gaussian noise, this probability is found as

$$
P_{i j}=\frac{1}{2} \operatorname{erfc}\left(\frac{I_{t h, j}-I_{i}}{\sigma_{i} \sqrt{2}}\right)-\frac{1}{2} \operatorname{erfc}\left(\frac{I_{t h, j+1}-I_{i}}{\sigma_{i} \sqrt{2}}\right) .
$$

Here $I_{i}$ denotes the photocurrent at symbol $i, I_{\mathrm{th}, j}$ is the threshold current, where $I_{\mathrm{th}, 0}=-\infty$, and $I_{\mathrm{th}, M}=+\infty$. The remaining decision thresholds are located between the subsequent symbols. The root mean square (RMS) value of the noise current at symbol $i$ is denoted by $\sigma_{i}$.

In this work, we consider equally spaced symbol levels $I_{i}$ and decision thresholds $I_{\mathrm{th}, j}$, so that $I_{i}=2 i I_{\mathrm{avg}} /(M-1)$ for $i=0, \ldots, M-1$ and $I_{\mathrm{th}, j}=(2 j-1) I_{\mathrm{avg}} /(M-1)$ for $j=$ $1, \ldots, M-1$, where $I_{\text {avg }}$ is the average photodetector current. Furthermore, the RMS noise currents $\sigma_{i}=\sigma$ are assumed to be the same for all symbols $i$. Substituted into (4), these assumptions yield

$$
\begin{aligned}
P_{i j}= & \frac{1}{2} \operatorname{erfc}\left(\frac{(2 j-2 i-1) I_{\mathrm{avg}}}{(M-1) \sigma \sqrt{2}}\right) \\
& -\frac{1}{2} \operatorname{erfc}\left(\frac{(2 j-2 i+1) I_{\mathrm{avg}}}{(M-1) \sigma \sqrt{2}}\right) .
\end{aligned}
$$

A common approximation at high signal-to-noise ratios and when the noise has a Gaussian distribution around the mean power level is to neglect all errors except those at minimum distance. Thus, setting $\operatorname{erfc}(x) \approx 0$ if $x \geq 3 I_{\mathrm{avg}} /((M-$ 1) $\sigma \sqrt{2})$ in (5) yields

$$
P_{i j} \approx \begin{cases}\frac{1}{2} \operatorname{erfc}\left(\frac{I_{\mathrm{avg}}}{(M-1) \sigma \sqrt{2}}\right), & |i-j|=1, \\ 0, & |i-j| \geq 2 .\end{cases}
$$

From (3) and (6) we finally obtain

$$
B E R \approx \frac{M-1}{M} \frac{d_{\mathrm{avg}}}{\log _{2} M} \operatorname{erfc}\left(\frac{I_{\mathrm{avg}}}{(M-1) \sigma \sqrt{2}}\right)
$$

where

$$
d_{\mathrm{avg}}=\frac{1}{M-1} \sum_{i=0}^{M-2} d_{i, i+1}
$$

is the average Hamming distance between the labels of adjacent symbols [15]. A comparison between the exact and approximate BER results was presented in [15] and the difference was shown to be negligible at low BER. 


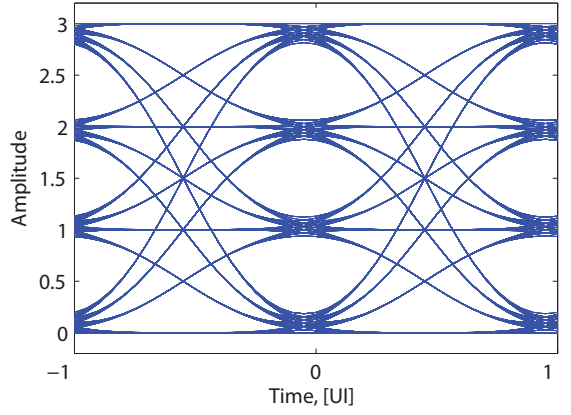

Fig. 1. Simulated 4-PAM eye diagram in a channel with Gaussian impulse response with decision thresholds in the middle between adjacent levels.

If Gray labeling is used, $d_{\text {avg }}=1$. If natural labeling is used it is [15]

$$
d_{\text {avg }}=2-\frac{\log _{2}(M)}{M-1} .
$$

It was shown in [15] that the performance difference between Gray and natural labeling is negligibly small in the low BER regime, which is of the most interest.

\section{B. Intersymbol Interference}

The ISI penalty due to the modal dispersion is well understood for OOK systems. The basic ISI penalty calculation methods were outlined in [19]. The worst case ISI penalty (expressed in $\mathrm{dB}$ ),

$$
P_{\mathrm{ISI}}=10 \log _{10}\left(\frac{1}{1-E_{m}}\right),
$$

where $E_{m}$ is the worst case relative eye closure. In case of OOK, it can be approximated with

$$
E_{m, \mathrm{OOK}}=1.425 \exp \left(-1.28\left(\frac{T}{T_{\mathrm{C}}}\right)^{2}\right) .
$$

The bit period is denoted as $T$ and the channel $10-90 \%$ risetime is denoted as $T_{\mathrm{C}}$. This ISI penalty calculation method, which is valid under assumption of Gaussian channel response and rectangular input pulse, was given with derivations in [20]. It is used in the IEEE 802.3 link budget spreadsheet [21]. Methods of calculation of the $10-90 \%$ rise-time for a given system are described in [20].

We can now extend the ISI penalty estimates to 4-PAM, assuming that it contains 3 stacked OOK eye diagrams. Assuming that the channel response is Gaussian, it is easy to observe that for the same system rise-time and symbol rate, the eye closure in case of 4-PAM is twice as large as in the case of OOK,

$$
E_{m, 4-\mathrm{PAM}}=2.85 \exp \left(-1.28\left(\frac{T}{T_{\mathrm{C}}}\right)^{2}\right) .
$$

For the considered channel response, the eye closures on all levels is the same, but the top and bottom eyes are asymmetric as shown in Fig. 1.

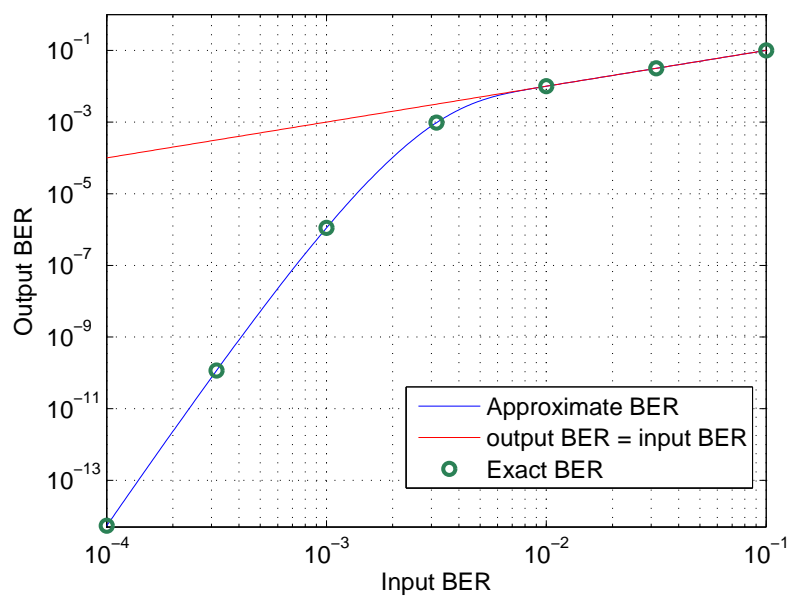

Fig. 2. Theoretical performance of Reed Solomon FEC with 255 symbols long codewords, 239 data symbols, eight-bit symbols, capable of correcting eight symbol errors. Approximate and exact result are show.

\section{Theoretical performance of Reed-Solomon FEC}

The Reed-Solomon (RS) codes, introduced in 1960 [22], are one of the most popular classes of error correcting codes, used in many communication technologies. Hardware implementations of RS codes have been studied and optimized over many years. The performance of RS FEC was investigated e.g. in [23].

The RS code with $n$-symbol-long codewords, each symbol of length $q$ bits, can correct $t=(n-k) / 2$ symbol errors, where $k$ is the number of payload data symbols. The code rate is $k / n$. For the common $\operatorname{RS}(255,239)$ code, $n=255$, $k=239, t=8$ and $q=8$. The rate is $239 / 255=0.94$ and the overhead is $16 / 239=6.7 \%$. The error rate at the output of the decoder can be calculated as a function of the bit-error rate at the input of the decoder. Assuming that the error probability at the input $B E R_{i n}$ is independent over all the bits in the FEC codeword, the incoming symbol error rate is

$$
S E R_{\text {in }}=1-\left(1-B E R_{\text {in }}\right)^{q} .
$$

Under assumptions that the decoder corrects all errors up to $t$ symbols and detects all errors above $t$ symbols, the symbol error rate at the output of the decoder is [24, Ch. 8]

$$
S E R_{\text {out }}=\frac{1}{n} \sum_{i=t+1}^{n} i\left(\begin{array}{c}
n \\
i
\end{array}\right) S E R_{\text {in }}^{i}\left(1-S E R_{\text {in }}\right)^{n-i} .
$$

TABLE I

INPUT VS. OUTPUT BER FOR RS $(255,239)$, SELECTED CASES OF INTEREST.

\begin{tabular}{|c|c|}
\hline Input BER & Output BER \\
\hline $1 \times 10^{-3}$ & $1.1 \times 10^{-6}$ \\
\hline $4.1 \times 10^{-4}$ & $1 \times 10^{-9}$ \\
\hline $1.8 \times 10^{-4}$ & $1 \times 10^{-12}$ \\
\hline
\end{tabular}




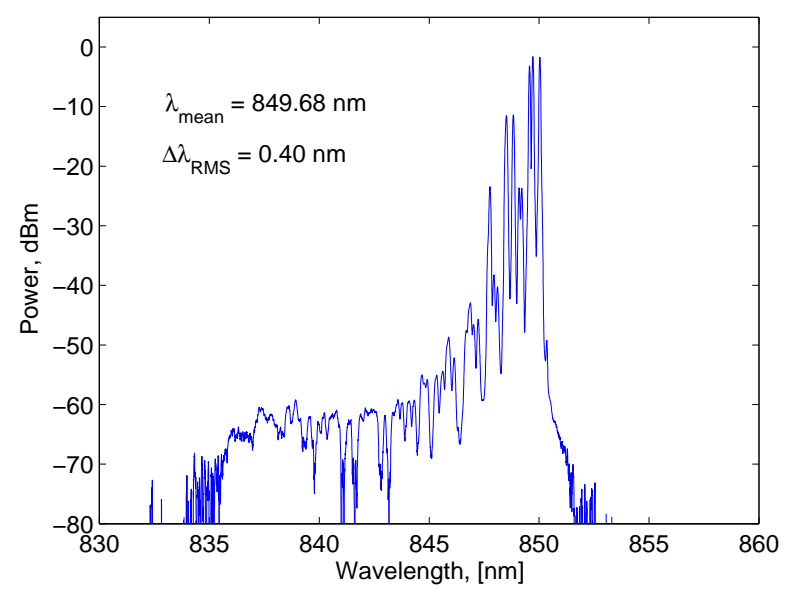

Fig. 3. Spectrum of the VCSEL used in the experiment at $8 \mathrm{~mA}$ bias current.

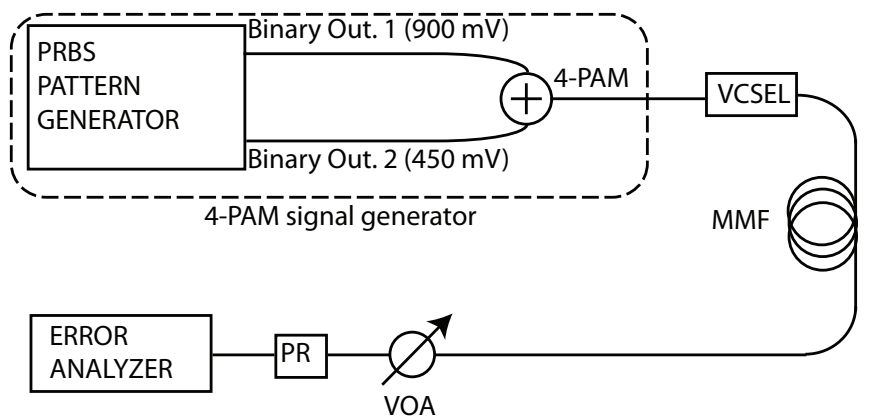

Fig. 4. Generalized experimental setup with 4-PAM signal generator.

The output bit-error rate can can be calculated from the output symbol error rate by inverting the step in (13),

$$
B E R_{\text {out }} \approx 1-\left(1-S E R_{\text {out }}\right)^{1 / q} .
$$

The last step is an approximation, the exact approach was given in [23]. In the Appendix we provide a simplified method of calculation of the exact BER, based on the results presented in [23].

The relation of the decoder output BER to the input BER for the $\operatorname{RS}(255,239)$ code is illustrated in Fig. 2, showing both the approximate results (15) and the exact results (26) obtained using method outlined in the Appendix. It is clear from Fig. 2 that the RS $(255,239)$ starts providing a significant improvement when input BER falls below $2 \times 10^{-3}$. Some of the interesting points of the relations between the input and output BER are listed in Table I. The difference between the exact and approximate output BER is less than $1 \%$. While the $\operatorname{RS}(255,239)$ is not the most powerful code available, it is used here as a benchmark, because of the popularity of the RS codes and well understood hardware implementations.

\section{EXPERIMENTAL SETUP}

The experiments were conducted with a directly modulated VCSEL with around $16 \mathrm{GHz}$ modulation bandwidth, OM4 MMF and an integrated photoreceiver with $10 \mathrm{GHz}-3 \mathrm{~dB}$ bandwidth. The laser spectrum at $8 \mathrm{~mA}$ bias current, which

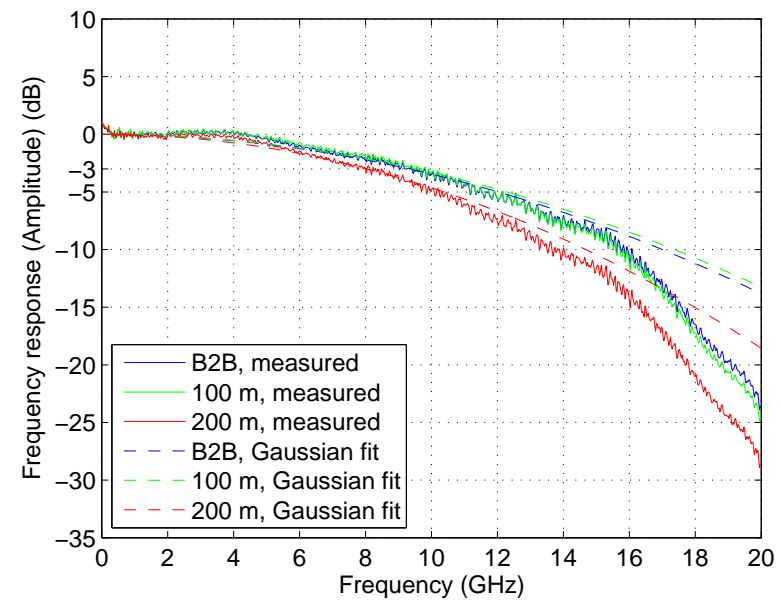

Fig. 5. Measured magnitude of the frequency response of the link, with Gaussian fits.

was kept constant for all experiments, is shown in Fig. 3 . The RMS spectral width was $0.40 \mathrm{~nm}$, which is less than the e.g. $0.65 \mathrm{~nm}$ requirement in [25, Sec. 6]. The light from the laser was coupled into a fiber through a lens package, with coupling optimized for maximum coupling efficiency. The launch conditions were set once at kept constant for all measurements. The following fiber lengths were tested: back to back (B2B), $100 \mathrm{~m}$, and $200 \mathrm{~m}$. A variable attenuator was placed before the photoreceiver to vary the received optical power. An overview of the setup is presented in Fig. 4. The 4-PAM signal was generated in real-time, by combination of two OOK signals, one of $900 \mathrm{mV}$ amplitude and the other of $450 \mathrm{mV}$. The OOK signals were offset in time to decorrelate them and achieve all transitions in the 4-PAM signal. This method of 4-PAM signal generation gives natural labeling of the symbols and was described in more detail in [26]. In all cases, the OOK bit streams were PRBS patterns of length $2^{7}-1$, which is often reported for high-speed VCSEL demonstrations [2], [12], [27], [28]. While longer patterns would certainly be interesting, the VCSEL in this experiment was driven through a Bias-T with $20 \mathrm{kHz}$ lower frequency cut-off, which has AC-coupled the modulation signal. A DCblock with $10 \mathrm{kHz}$ lower frequency cut-off was used after the photoreciever. In practical applications various line codes are used to provide adequate clock recovery and bias neutrality [29], [30]. The BER was measured in real-time using an error analyzer. The pattern generator and error analyzer were driven from the same external clock source, as shown in Fig. 4. The real-time BER measurement method for 4-PAM was described

TABLE II

MEASURED -3 DB BANDWIDTH FOR THE TESTED FIBER LENGTHS.

\begin{tabular}{|c|c|}
\hline length $[\mathrm{m}]$ & $-3 \mathrm{~dB}$ BW $[\mathrm{GHz}]$ \\
\hline 0 & 9.5 \\
\hline 100 & 9.4 \\
\hline 200 & 8.3 \\
\hline
\end{tabular}




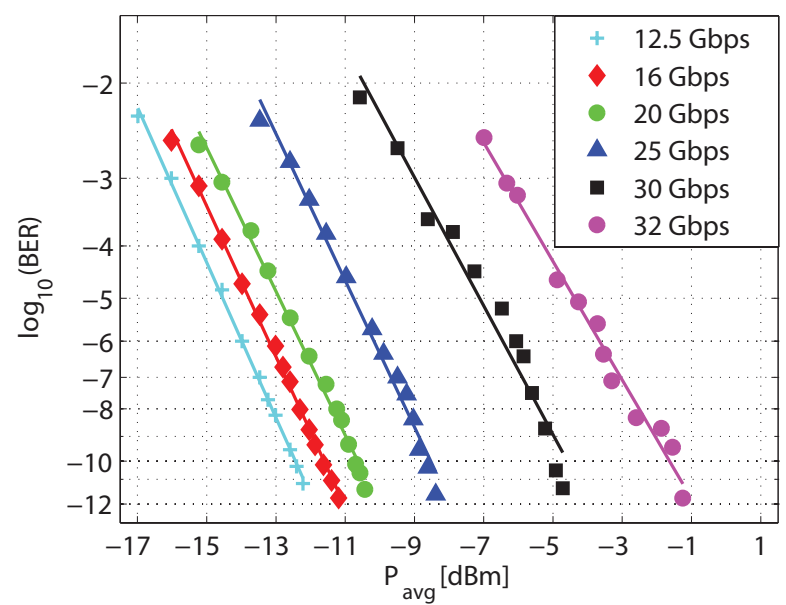

Fig. 6. Measured BER for OOK in B2B configuration.

in detail also in [26]. In short, the total BER was derived from the error rates measured between the adjacent 4-PAM signal levels. If the error rates measured at these thresholds are denoted $E R_{1}, E R_{2}, E R_{3}$, then the total BER is given by [26]

$$
B E R=\frac{1}{2} E R_{1}+E R_{2}+\frac{1}{2} E R_{3} .
$$

It is assumed, similarly to Sec. II-C, that the error probabilities are independent over all the bits. The threshold levels were optimized individually for each eye, since the experimental setup did not include an automatic gain control (AGC) circuit. The sampling time for all thresholds was the same and optimized from the center eye. The BER was measured only at the optimal timing and timing jitter is not analyzed in this work.

The magnitude of the frequency response of the system was measured for each tested fiber length with a vector network analyzer and the results are presented in Fig. 5, together with Gaussian fits. The measured phase responses were linear for all cases. All fiber lengths were compared at the same launch conditions. The measured $-3 \mathrm{~dB}$ bandwidths are illustrated in Table II. It is evident from Fig. 5 that the Gaussian fits correspond well to the measured frequency responses up around $16 \mathrm{GHz}$, but they underestimate the roll-off at higher frequencies. The signal loss at $16 \mathrm{GHz}$ is around $-10 \mathrm{~dB}$, and thus the amount of signal power in the frequency range where the Gaussian model is not adequate is small.

\section{EXPERIMENTAL RESULTS}

\section{A. BER results}

In order to investigate the ISI performance of 4-PAM, and to compare it with OOK, for each fiber length and modulation format, a range of data rates were tested. The BER was measured down to roughly $10^{-12}$. The BER results for OOK and 4-PAM are illustrated in Figs. 6-11. The BER is plotted against average received optical power, uncorrected for the $10 \mathrm{~dB}$ extinction ratio. Apparently, at lower data rates, the measured points follow better the fit lines. This is probably

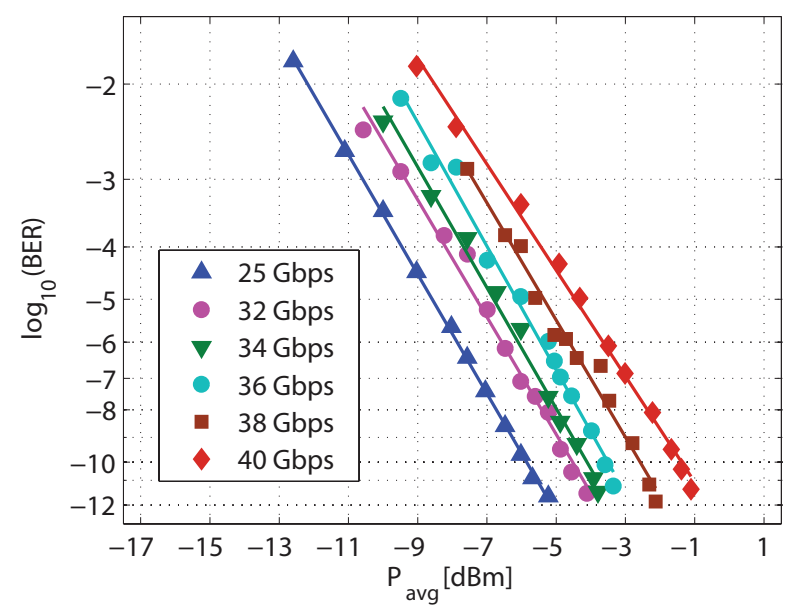

Fig. 7. Measured BER for 4-PAM in B2B configuration.

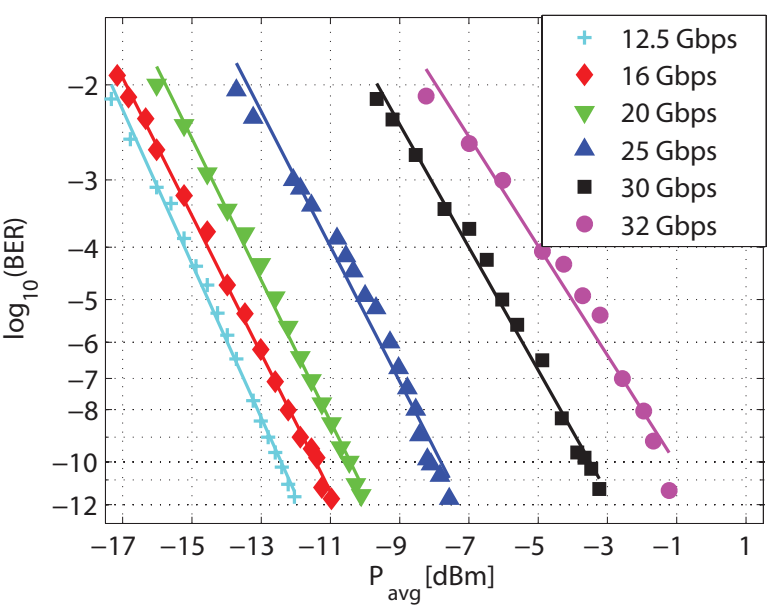

Fig. 8. Measured BER for OOK after transmission through $100 \mathrm{~m}$ of OM4 fiber.

because the optimization of the decision point was more difficult at higher data rates and with larger ISI effects.

The experimental BER was used to extract the receiver sensitivity at BER of $10^{-12}$ for each fiber length. To investigate a hypothetical use of FEC, we took the $\operatorname{RS}(255,239)$ code with $7 \%$ overhead, described in II-C. A BER threshold of $1.8 \times 10^{-4}$ was assumed, because it would yield output BER of $10^{-12}$ (see Table I), for comparison with the uncoded sensitivity. The extracted sensitivities at various data rates are illustrated in Figs. 12-14 for the fiber lengths from B2B to $200 \mathrm{~m}$, respectively. The figures include also theoretical sensitivity at BER of $10^{-12}$, with ISI penalties for each case. The theoretical sensitivity was calculated using the theoretical BER expressions outlined in section II-A and the ISI penalties, using expressions outlined in section II-B. Example eye diagrams for high data rate OOK and 4-PAM are included as insets in Figs. 12-14. Fiber bending was not investigated rigorously, but it did not seem to have noticeable impact on system performance. 


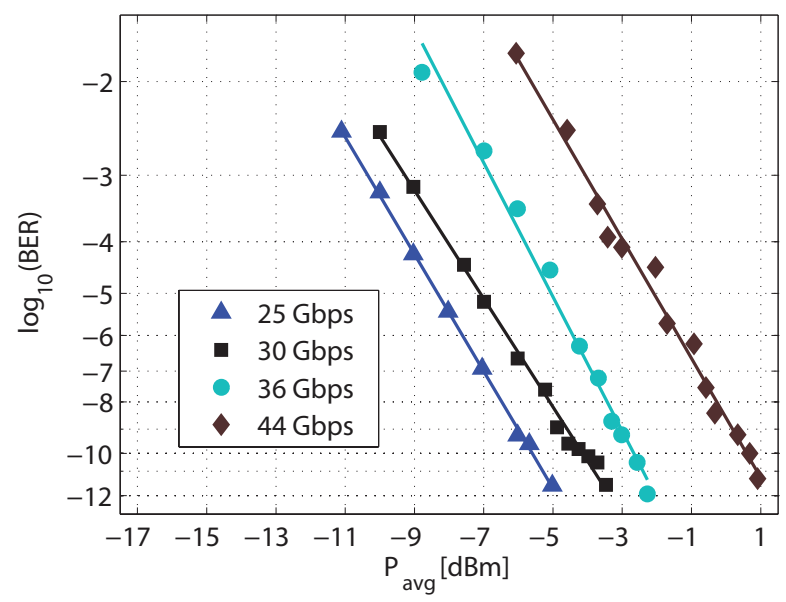

Fig. 9. Measured BER for 4-PAM after transmission through $100 \mathrm{~m}$ of OM4 fiber.

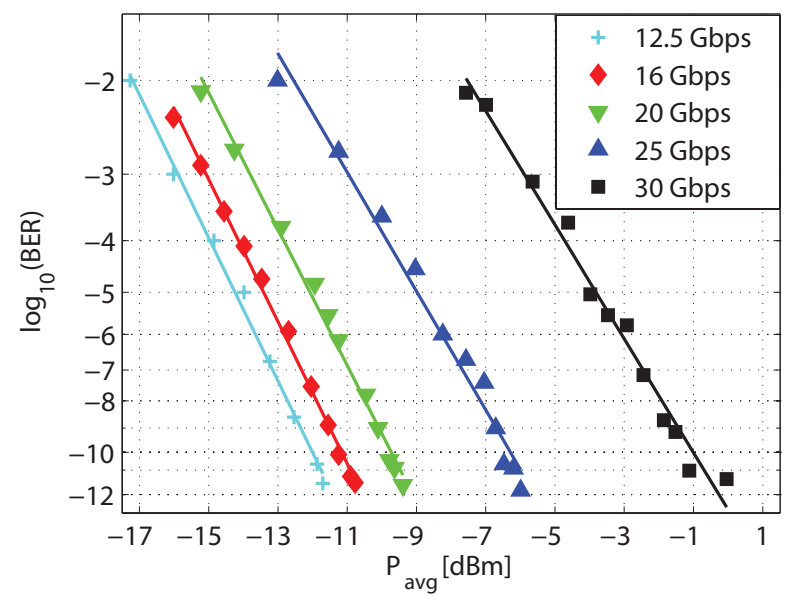

Fig. 10. Measured BER for OOK after transmission through $200 \mathrm{~m}$ of OM4 fiber.

\section{B. Discussion}

The fastest achieved data rate with BER down to $10^{-12}$ in the presented experiments is 44 Gbps, over $100 \mathrm{~m}$ of OM4type MMF with 4-PAM modulation. This is comparable to the state of the art result with OOK presented in [31], where 44 Gbps transmission over $50 \mathrm{~m}$ of the same type OM4 MMF was demonstrated, using a high speed VCSEL and a photoreceiver. The sensitivity at this bit rate was around 0 $\mathrm{dBm}$. For comparison, in [1], OOK was demonstrated at 44 Gbps in BTB configuration with similar sensitivity, using a high speed VCSEL with $28 \mathrm{GHz}$ bandwidth; however, in that work a photodetector without matched integrated amplifier was used. In general, the maximum ISI-limited data rates obtained with 4-PAM were around 4 times the $-3 \mathrm{~dB}$ bandwidth of the system. It is clear from the eye diagrams in the inserts in Figs. 12-14 that the highest data rate results offer no margins, as the eyes are almost closed. Thus, they present the limiting data rates for the tested system.

It is clear from the sensitivity plots in Figs. 12-14 that the ISI model works very well for OOK, with very small

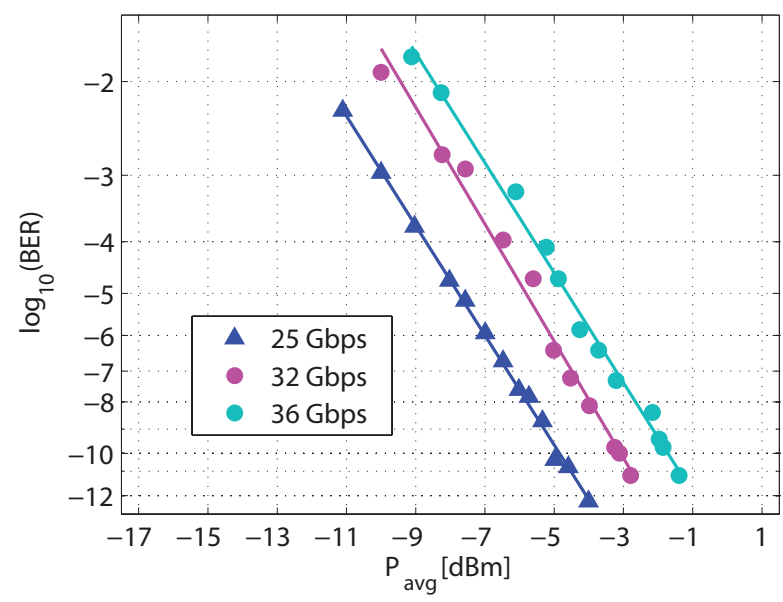

Fig. 11. Measured BER for 4-PAM after transmission through $200 \mathrm{~m}$ of OM4 fiber.

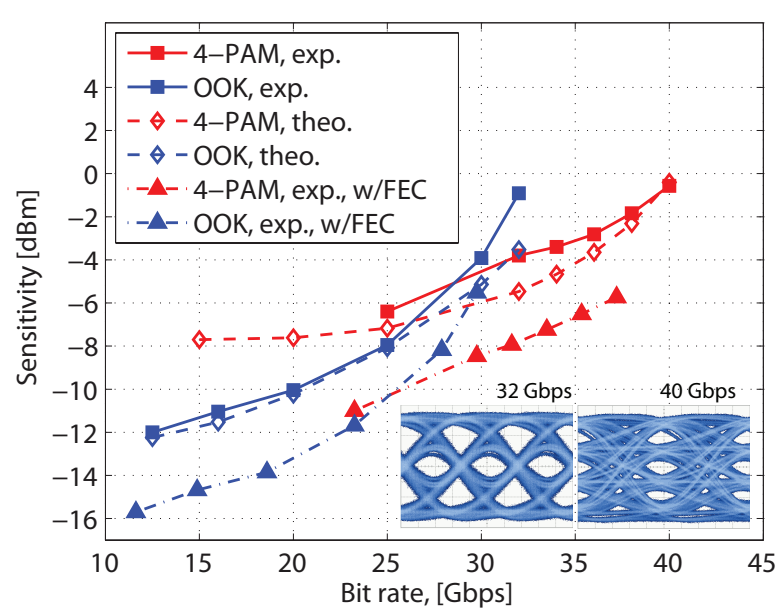

Fig. 12. Measured sensitivity at $B E R=10^{-12}$, theoretical sensitivity at $\mathrm{BER}=10^{-9}$ and measured sensitivity at $\mathrm{BER}=1.8 \times 10^{-4}$, with data rates reduced by the $7 \%$ FEC overhead, measured in the $\mathrm{B} 2 \mathrm{~B}$ setting.

deviations between the experimental and theoretical results. The matters look similar in case of 4-PAM. The ISI penalty increases with increased ratio of the bit rate to the channel bandwidth in a similar manner as in the case of OOK, however the theoretical and experimental results agree less. The theoretical sensitivity degradation by the ISI effects is uniformly underestimated at low bit rates and overestimated at high bit rates. Simple simulations have shown that in case of 4-PAM, the observed level of discrepancy (up to $2 \mathrm{~dB}$ ) can be caused by deviations of the pulse shape of from the ideal Gaussian shape. Accurate calculation of the ISI penalties in case of 4-PAM would require use of exact pulse shape and channel impulse response, but a rough investigation could be done with the simple theoretical expression given in Section II-B.

The experiments and theory show that there are cases when 4-PAM offers advantages over OOK, at least from the perspective of power penalties. When the received optical power was above $-5 \mathrm{dBm}$, 4-PAM yielded higher throughput than OOK, in the case without FEC. Theoretically, with 


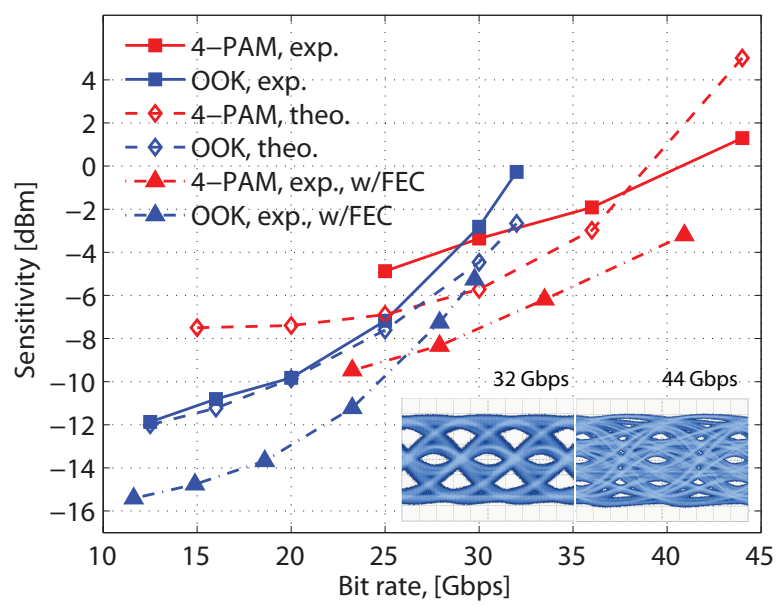

Fig. 13. The same sensitivities as in Fig. 12, after transmission of $100 \mathrm{~m}$ of OM4-type MMF.

application of FEC, 4-PAM becomes better than OOK when the received optical power is above $-10 \mathrm{dBm}$. A theoretical application of FEC to the experimental BER results shows that the FEC can increase the link throughput at a given receiver power level, or improve the sensitivity at a given bit rate. The sensitivity improvement can be as large as $4 \mathrm{~dB}$, which is quite substantial. It brings the sensitivity of 4-PAM at $25 \mathrm{Gbps}$ down to $-10 \mathrm{dBm}$ in the $\mathrm{B} 2 \mathrm{~B}$ case. It must be noted here that the theoretical FEC performance given in Sec. II-C is valid only for independent bit errors. This is not the case when ISI is introduced. Therefore, when the ISI penalty is introduced, the theoretical prediction of the FEC performance becomes less accurate.

The link budget foreseen in the IEEE 802.3ba standard is $8.3 \mathrm{dBm}$. With input power into the link of around $0 \mathrm{dBm}$, 4-PAM with FEC becomes comparable with OOK at $25 \mathrm{Gbps}$, while still having a considerable margin for ISI, while OOK reaches its limits in the presented system. On the other hand, cost, power consumption of the electronics, and latency due to the FEC would have to be thoroughly investigated. When it comes to complexity of the 4-PAM circuits, some insight can be gained from results presented in [32]. The highest available system throughput could be achieved if the link was managed in a best-effort fashion, when the modulation format would be adapted to the received optical power. On the other hand, many standards links (e.g. IEEE 802.3ba or FibreChannel) use fixed data rate links. In this case, the modulation format has to be chosen to accommodate the worst-case scenario for the link budget.

The experimental results suggest that for data rates comparable to the channel bandwidth, OOK is the best choice, since it offers the best sensitivity and simplicity. If higher spectral efficiency is needed, 4-PAM with FEC would be a good choice from the sensitivity point of view, since it can double the data rates, with only small increase in required received optical power.

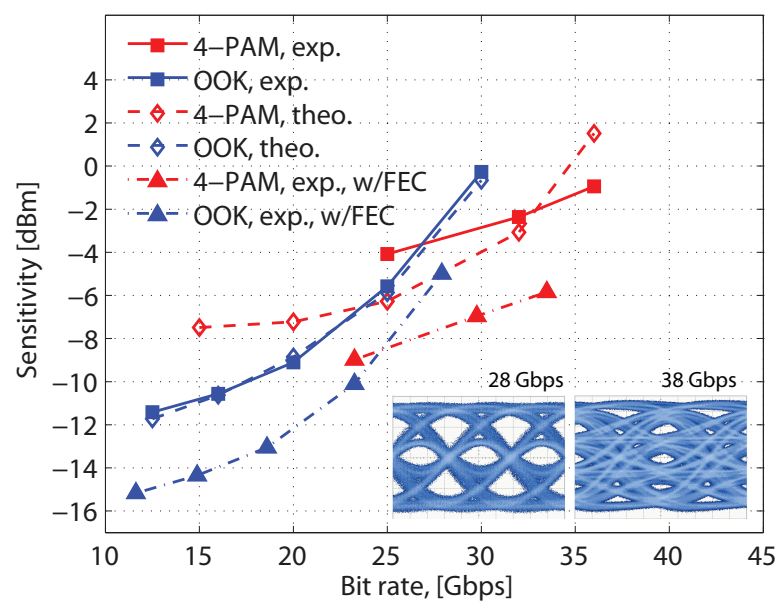

Fig. 14. The same sensitivities as in Fig. 12, after transmission of $200 \mathrm{~m}$ of OM4-type MMF.

\section{CONCLUSIONS}

The $-3 \mathrm{~dB}$ bandwidth of the tested link was below $10 \mathrm{GHz}$, limited by the photoreceiver. The maximum achieved data rate with 4-PAM was $44 \mathrm{Gbps}$, over $100 \mathrm{~m}$ of OM4-type MMF at $0 \mathrm{dBm}$ received optical power. Maximum achieved data rate with OOK was $32 \mathrm{Gbps}$ over the same distance.

We have compared OOK and 4-PAM at increasing data rates in a system with fixed bandwidth. As expected, 4-PAM yielded higher maximum data throughput than OOK, at the cost of higher required received power.

It was also confirmed that the Gaussian model of the impulse response and signal pulse shape gives very good results for OOK, but deviations of up to $2 \mathrm{~dB}$ were present in case of 4-PAM and that if more accurate calculation of ISI penalties for 4-PAM is needed, actual pulse shape should be used.

The use of FEC with 4-PAM could yield up to $4 \mathrm{~dB}$ improvement in sensitivity at a given bit rate, bringing the sensitivity of 25 Gbps 4-PAM down to $-10 \mathrm{dBm}$, in a link with almost $10 \mathrm{GHz}$ bandwidth. This sensitivity would be acceptable in practical applications, given the power budgets in today's standards defining short-range optical links. If other aspects like power consumption of the FEC electronics and added latency would not turn out to be limiting factors, introduction of FEC could enable application of the 4-PAM in short-range optical communication.

If a faster photoreceiver was used, the frequency response of the system, at least in the BTB case would be dominated by the shape of the frequency response of the VCSEL. We expect, that if that response was flat up to the resonance frequency, and then had a fast roll-off the relative performance of 4PAM and OOK would be similar as in the case of Gaussian frequency response. Another aspect of comparison, which was not addressed here is comparison of timing margins for 4-PAM and OOK with the types of frequency response observed in VCSEL based optical links. Some jitter measurement results for 4-PAM transmission are presented in [33]. 


\section{APPENDIX}

\section{Calculation of The outPut BER of ReEd-Solomon CODE}

An accurate way of calculation of the output BER as a function of the input BER for a RS FEC was outlined in [23]. Here, we present a simplified method of the output BER calculation, suitable for a computer implementation. The output BER is given by

$$
B E R_{\mathrm{out}}=\sum_{j=t+1}^{n} \sum_{i=j}^{q j} P\left(\epsilon \mid w_{i}, W, j\right) P\left(W_{j} \mid w_{i}\right) P\left(w_{i}\right),
$$

where $w_{i}$ denotes the event of $i$ bit errors in the received codeword, $W_{j}$ denotes the event of $j$ symbol errors in the received codeword and $\epsilon$ denotes a bit error in the received codeword. The factors of (17) were given in [23] and it can be represented as

$$
\begin{array}{r}
B E R_{\text {out }}=\sum_{j=t+1}^{n}\left(\begin{array}{c}
n \\
j
\end{array}\right) \sum_{i=j}^{q j} \frac{i}{q n} B E R_{\mathrm{in}}{ }^{i}\left(1-B E R_{\mathrm{in}}\right)^{q n-i} \\
\sum_{m=0}^{\lfloor j-i / q\rfloor}(-1)^{m}\left(\begin{array}{c}
j \\
m
\end{array}\right)\left(\begin{array}{c}
q(j-m) \\
i
\end{array}\right),
\end{array}
$$

where $B E R_{\text {in }}$ is the input BER. This equation can be expressed in a simpler form as

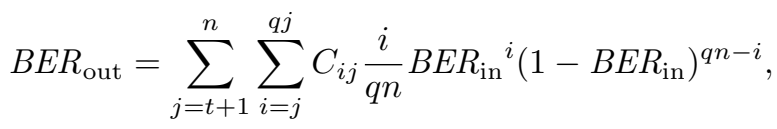

where

$$
C_{i j}=\left(\begin{array}{c}
n \\
j
\end{array}\right) \sum_{m=0}^{j-\lceil i / q\rceil}(-1)^{m}\left(\begin{array}{c}
j \\
m
\end{array}\right)\left(\begin{array}{c}
q(j-m) \\
i
\end{array}\right)
$$

The $C_{i j}$ coefficients are integers, and do not depend on $B E R_{\text {in }}$ or $t$. They can be tabulated offline for given $n$ and $q$. The calculations can be further simplified by using the relation

$$
\sum_{j=\lceil i / q\rceil}^{\min (n, i)} C_{i j}=\left(\begin{array}{c}
q n \\
i
\end{array}\right),
$$

for $i=1, \ldots, q n$, and because, with the definition of $P\left(W_{j} \mid w_{i}\right)$ given in [23]

$$
\sum_{j=\lceil i / q\rceil}^{\min (n, i)} P\left(W_{j} \mid w_{i}\right)=1 .
$$

We can interchange the order of summation in (19),

$$
t+1 \leq j \leq n \wedge j \leq i \leq q j \Longleftrightarrow j_{o} \leq j \leq j_{1},
$$

where

$$
\left\{\begin{array}{l}
j_{0} \equiv \max \left(t+1,\left\lceil\frac{i}{q}\right\rceil\right) \\
j_{1} \equiv \max (n, i)
\end{array}\right.
$$

From $j_{o} \leq j_{1}$ follows

$$
t+1 \leq i \leq q n .
$$

Thus, (19) becomes

$$
B E R_{\mathrm{out}}=\sum_{i=t+1}^{q n} E_{t}(i) \frac{i}{q n} B E R_{\mathrm{in}}^{i}\left(1-B E R_{\mathrm{in}}\right)^{q n-i},
$$

where

$$
E_{t}(i) \equiv \sum_{j=j_{0}}^{j_{1}} C_{i j}
$$

depends on $t$ but not $p$. From (21) follows

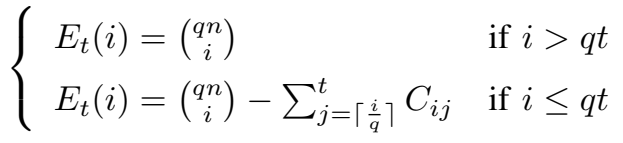

because $t+1 \leq\lceil i / q\rceil \Longleftrightarrow i \geq q t+1$. The final strategy to evaluate $P_{E}$ is thus:

1) for given $n$ and $q$, tabulate $C_{i j}$ from (20), offline with high accuracy, for $j=1, \ldots, t$ and $i=j, \ldots, q j$.

2) For a given $t$, tabulate $E_{t}(i)$ from (28) for $i=t+$ $1, \ldots, q n$.

With the definition in (27), the $E_{t}(i)$ has the following properties:

- $E_{t}(i)=0$ if $1 \leq i \leq t$,

- $E_{t}(i)=\left(\begin{array}{c}q n \\ i\end{array}\right)$ if $q t \leq i \leq q n$.

Thus, it is sufficient to tabulate $E_{t}(i)$ for $i=t+1, \ldots, q t$.

\section{REFERENCES}

[1] P. Westbergh, R. Safaisini, E. Haglund, B. Kögel, J. S. Gustavsson, A. Larsson, M. Geen, R. Lawrence, and A. Joel, "High-speed $850 \mathrm{~nm}$ VCSELs with $28 \mathrm{GHz}$ modulation bandwidth operating error-free up to 44 Gbit/s," Electron. Lett., vol. 48, no. 18, pp. 1145-1147, Aug. 2012.

[2] W. Hofmann, P. Moser, P. Wolf, A. Mutig, M. Kroh, and D. Bimberg, "44 Gb/s VCSEL for optical interconnects," in Proc. OFC, Mar. 2011, p. PDPC5.

[3] R. Safaisini, S. K., E. Haglund, P. Westbergh, J. S. Gustavsson, A. Larsson, and P. A. Andrekson, "20 Gbit/s error-free operation of $850 \mathrm{~nm}$ oxide-confined vcsels beyond $1 \mathrm{~km}$ of multimode fibre," Electron. Lett., vol. 48 , no. 19 , pp. 1225-1227, Sept. 2012.

[4] K. Szczerba, B. Olsson, P. Westbergh, A. Rhodin, J. S. Gustavsson, Å. Haglund, M. Karlsson, A. Larsson, and P. A. Andrekson, "37 Gbps transmission over $200 \mathrm{~m}$ of MMF using single cycle subcarrier modulation and a VCSEL with $20 \mathrm{GHz}$ modulation bandwidth," in Proc. ECOC, Sept. 2010, p. We.7.B.2.

[5] S. C. J. Lee, F. Breyer, S. Randel, D. Cardenas, H. P. A. van den Boom, and A. M. J. Koonen, "Discrete multitone modulation for high-speed data transmission over multimode fibers using 850-nm VCSEL," in Proc. OFC, Mar. 2009, p. OWM2.

[6] S. Randel, F. Breyer, and S. C. J. Lee, "High-speed transmission over multimode optical fibers," in Proc. OFC, Feb. 2008, p. OWR2.

[7] J. M. Kahn and J. R. Barry, "Wireless infrared communications," Proc. IEEE, vol. 85, no. 2, pp. 265-298, Feb. 1997.

[8] D. Watanabe, A. Ono, and T. Okayasu, "CMOS optical 4-PAM VCSEL driver with modal-dispersion equalizer for $10 \mathrm{~Gb} / \mathrm{s} 500 \mathrm{~m}$ MMF transmission," in Proc. ISSCC, Feb. 2009, pp. 106-107.

[9] T. Toifl, C. Menolfi, M. Ruegg, R. Reutemann, P. Buchmann, M. Kossel, T. Morf, J. Weiss, and M. L. Schmatz, "A 22 Gb/s PAM-4 receiver in 90-nm CMOS SOI technology," IEEE J. Solid-State Circuits, vol. 41, no. 4, pp. 954-965, April 2006.

[10] S. Walklin and J. Conradi, "Multilevel signaling for increasing the reach of $10 \mathrm{~Gb} / \mathrm{s}$ lightwave systems," IEEE J. Lightw. Technol., vol. 17, no. 11, pp. 2235-2248, Nov. 1999 .

[11] J. D. Ingham, R. V. Penty, and I. H. White, "10 Gb/s \& $20 \mathrm{~Gb} / \mathrm{s}$ extended-reach multimode-fiber datacommunication links using multilevel modulation and transmitter-based equalization," in Proc. OFC, Feb. 2008, p. OTuO7. 
[12] J. D. Ingham, R. V. Penty, I. H. White, P. Westbergh, J. S. Gustavsson, A. Haglund, and A. Larsson, " $32 \mathrm{~Gb} / \mathrm{s}$ multilevel modulation of an 850 $\mathrm{nm}$ VCSEL for next-generation datacommunication standards," in Proc. CLEO, May 2011, p. CWJ2.

[13] J. E. Cunningham, D. Beckman, X. Zheng, D. Huang, T. Sze, and A. V. Krishnamoorthy, "PAM-4 signaling over VCSELs with $0.13 \mu \mathrm{m}$ CMOS chip technology," Opt. Express, vol. 14, no. 25, pp. 12028-12 038, Dec. 2006.

[14] K. Szczerba, P. Westbergh, J. S. Gustavsson, Å. Haglund, J. Karout, M. Karlsson, P. Andrekson, E. Agrell, and A. Larsson, "30 Gbps 4-PAM transmission over $200 \mathrm{~m}$ of MMF using a VCSEL," in Proc. ECOC, Sept. 2011, p. Tu.3.C.

[15] K. Szczerba, P. Westbergh, J. Karout, J. S. Gustavsson, Å. Haglund, M. Karlsson, P. A. Andrekson, E. Agrell, and A. Larsson, "4-PAM for high-speed short-range optical communications," IEEE/OSA J. Opt. Commun. Netw, vol. 4, no. 11, pp. 885-894, Nov. 2012.

[16] D. Kam, M. Ritter, T. Beukema, J. Bulzacchelli, P. Pepeljugoski, Y. Kwark, L. Shan, X. Gu, C. Baks, R. John, G. Hougham, C. Schuster, R. Rimolo-Donadio, and B. Wu, "Is $25 \mathrm{gb} / \mathrm{s}$ on-board signaling viable?" IEEE Trans. Adv. Packag, vol. 32, no. 2, pp. 328-344, 2009.

[17] J. K. Pollard, "Multilevel data communication over optical fibre," IEE Proc.-Commun., vol. 138, no. 3, pp. 162-168, Jun. 1991.

[18] J. Proakis and M. Salehi, Digital communications. McGraw-Hill Higher Education, 2008.

[19] J. Gimlett and N. Cheung, "Dispersion penalty analysis for LED/singlemode fiber transmission systems," IEEE J. Lightw. Technol., vol. 4, no. 9, pp. 1381-1392, Sept. 1986.

[20] D. Cunningham, M. Nowell, D. Hanson, and L. Kazovsky, "The IEEE $802.3 \mathrm{z}$ worst case link model for optical physical media dependent specification," 1997. [Online]. Available: http://www.ieee802.org/3/z/ public/presentations/mar1997/DCwpaper.pdf

[21] "IEEE 802.3ae 10G Ethernet optical link budget spreadsheet," 2000 [Online]. Available: http://ieee802.org/3/10G_study/public/email_attach/ All_1250v2.xls

[22] I. Reed and G. Solomon, "Polynomial codes over certain finite fields," J. Soc. Ind. Appl. Math., vol. 8, no. 2, pp. 300-304, Jun. 1960.

[23] W. Ebel and W. Tranter, "The performance of Reed-Solomon codes on a bursty-noise channel," IEEE Trans. Commun., vol. 43, no. 234, pp. 298-306, Feb./Mar./Apr. 1995.

[24] B. Sklar, Digital communications: fundamentals and applications. Prentice-Hall PTR, 2001

[25] IEEE Standard for Ethernet, 2012, no. IEEE Standard 802.3-2012.

[26] K. Szczerba, P. Westbergh, J. Karout, J. S. Gustavsson, Å. Haglund, M. Karlsson, P. A. Andrekson, E. Agrell, and A. Larsson, "30 Gbps 4-PAM transmission over $200 \mathrm{~m}$ of MMF using an $850 \mathrm{~nm}$ VCSEL," Opt. Express, vol. 19, no. 26, pp. 203-208, Dec. 2011.

[27] S. Blokhin, J. Lott, A. Mutig, G. Fiol, N. Ledentsov, M. Maximov, A. Nadtochiy, V. Shchukin, and D. Bimberg, "Oxide-confined $850 \mathrm{~nm}$ VCSELs operating at bit rates up to $40 \mathrm{Gbit} / \mathrm{s}$," Electron. Lett., vol. 45, no. 10 , pp. 501-503, 2009.

[28] J. D. Ingham, R. V. Penty, I. H. White, and D. G. Cunningham, "40 $\mathrm{gb} / \mathrm{s}$ carrierless amplitude and phase modulation for low-cost optical datacommunication links," in Proc. OFC, 2011, p. OThZ3.

[29] H. Frazier, "The 802.3z Gigabit Ethernet standard," Network, IEEE, vol. 12, no. 3, pp. 6-7, May/Jun. 1998.

[30] IEEE Standard for Information technology Telecommunications and information exchange between systems Local and metropolitan area networks Specific requirements, 2010, no. IEEE Standard 802.3ba.

[31] P. Westbergh, R. Safaisini, E. Haglund, J. Gustavsson, A. Larsson, M. Geen, R. Lawrence, and A. Joel, "High-speed oxide confined 850$\mathrm{nm}$ VCSELs operating error-free at $40 \mathrm{~Gb} / \mathrm{s}$ up to $85^{\circ} \mathrm{c}$," IEEE Photon. Technol. Lett., vol. 25, no. 8, pp. 768-771, 2013.

[32] J. Lee, M.-S. Chen, and H.-D. Wang, "Design and comparison of three 20-Gb/s backplane transceivers for duobinary, PAM4, and NRZ data," IEEE J. Solid-State Circuits, vol. 43, no. 9, pp. 2120-2133, 2008

[33] K. Szczerba, P. Westbergh, M. Karlsson, A. P. A., and A. Larsson, "60 Gbits error-free 4-PAM operation with $850 \mathrm{~nm}$ VCSEL," Electron. Lett., vol. 49 , no. 15 , pp. $953-55$, July 2013

Krzysztof Szczerba has received the M.Sc. degree in telecommunications from Technical University of Denmark, Lyngby, Denmark, and an M.Sc. degree in electronics and telecommunications from Technical University of Lodz, Poland, both in 2008. In 2008 he worked at Technical University of
Denmark, DTU Fotonik, Department of Photonics Engineering as a research assistant. In 2009 he joined the Chalmers University of Technology, Department of Microtechnology and Nanoscience in Gothenburg, Sweden, where he is currently working towards the Ph.D. degree in optical communications. His research interest are focused on modulation formats for short range optical links for datacomm applications.

Petter Westbergh received the M.Sc. degree in Engineering Physics and the Ph.D. degree in Microtechnology and Nanoscience from Chalmers University of Technology, Göteborg, Sweden, in 2007 and 2011, respectively. His thesis focused on the design, fabrication, and characterization of high speed $850 \mathrm{~nm}$ vertical cavity surface emitting lasers (VCSELs) intended for application in short reach communication networks. He is currently continuing his work on improving the performance of high speed VCSELs with the department of Microtechnology and Nanoscience at Chalmers University of Technology.

Erik Agrell received the M.S. degree in electrical engineering in 1989 and the $\mathrm{Ph} . \mathrm{D}$. degree in information theory in 1997, both from Chalmers University of Technology, Sweden.

From 1988 to 1990, he was with Volvo Technical Development as a Systems Analyst, and from 1990 to 1997, with the Department of Information Theory, Chalmers University of Technology, as a Research Assistant. In 19971999, he was a Postdoctoral Researcher with the University of Illinois at Urbana-Champaign and the University of California, San Diego. In 1999, he joined the faculty of Chalmers University of Technology, first as an Associate Professor and since 2009 as a Professor in Communication Systems. His research interests belong to the fields of information theory, coding theory, and digital communications, and his favorite applications are found in optical communications. More specifically, his current research includes bitinterleaved coded modulation and multilevel coding, polarization-multiplexed coding and modulation, transmission over the optical intensity channel, phase noise mitigation, and bit-to-symbol mappings in coded and uncoded systems.

Magnus Karlsson received his Ph.D. in 1994 from Chalmers University of Technology, Gothenburg, Sweden. Since 1995, he has been with the Photonics Laboratory at Chalmers, first as assistant professor and since 2003 as professor in photonics. He has authored or co-authored over 200 scientific journal and conference contributions and is currently associate editor of Optics Express. He has served in the technical committee for the Optical Fiber Communication Conference (OFC), and currently serves in the technical program committees for the European Conference of Optical Communication (ECOC), and the Asia Communications and Photonics Conference (ACP). He was appointed Fellow of the Optical Society of America in 2012. His research has been devoted to mainly nonlinear fiber optics and optical communications in a broad sense, from fundamental transmission effects such as fiber nonlinearities and polarization mode dispersion, to applied issues such as high-capacity data transmission and all-optical switching. Currently he works on transmission impairments and multilevel modulation formats for intensity-modulated and coherent fiber optic links. 
Peter A. Andrekson received his Ph.D. from Chalmers University of Technology, Sweden, in 1988. After about three years with AT\&T Bell Laboratories, Murray Hill, N.J., USA, during 1989-1992, he returned to Chalmers where he is now a full professor at the Department of Microtechnology and Nanoscience. He was Director of Research at Cenix Inc. in Allentown, PA, USA, during 2000-2003 and with the newly established Center for Optical Technologies at Lehigh University, Bethlehem, PA, USA during 2003-2004. His research interests include nearly all aspects of fiber communications such as optical amplifiers, nonlinear pulse propagation, all-optical functionalities, and very high capacity transmission. He is co-founder of the optical test \& measurement company Picosolve Inc., now part of EXFO and where Andrekson is Director of EXFO Sweden AB.

Andrekson is a Fellow of the Optical Society of America and a Fellow of the IEEE. He is the author of about three hundred fifty scientific publications and conference papers in the area of optical communications, among which eighty were invited papers at leading international conferences and journals, including two tutorials at the Optical Fiber Communication Conference (OFC) in 2004 and 2011. He is an elected member of the Board of Governors for the IEEE Photonics Society and is or has served on several technical program committees, including OFC and ECOC, and as international project and candidate evaluator, and has also twice served as an expert for the evaluation of the Nobel Prize in Physics. He was an associate editor for IEEE Photonics Technology Letters during 2003-2007. In 1993 he was awarded a prize from the Swedish government research committee for outstanding work performed by young scientists, and in 2000 he was awarded the Telenor Nordic research award for his contribution to optical technologies.

Anders Larlsson received the M.Sc. and Ph.D. degrees in electrical engineering from Chalmers University of Technology, Göteborg, Sweden, in 1982 and 1987, respectively. In 1991, he joined the faculty at Chalmers University of Technology where he was promoted to Professor in 1994. From 1984 to 1985 he was with the Department of Applied Physics, California Institute of Technology, and from 1988 to 1991 with the Jet Propulsion Laboratory, both at Pasadena, CA, USA. He has been a guest professor at Ulm University (Germany), at the Optical Science Center, University of Arizona, Tucson, Arizona (USA), at Osaka University (Japan), and at the Institute of Semiconductors, Chinese Academy of Sciences (China). He coorganized the IEEE Semiconductor Laser Workshop 2004, organized the European Semiconductor Laser Workshop 2004, and was the Program and General Chair for the IEEE International Semiconductor Laser Conference in 2006 and 2008, respectively. He is an associate editor for IEEE Journal of Lightwave Technology. His scientific background is in the areas of materials and devices for optical communication, information processing, and sensing. Currently, his research is focused on vertical cavity surface emitting lasers, optically pumped semiconductor disk lasers, and III-nitride emitters. He has published more than 400 scientific journal and conference papers. He is a Fellow of the European Optical Society and a Senior Member of IEEE. 